\title{
Transcatheter therapies for secondary mitral regurgitation in advanced heart failure: what are we aiming for?
}

\author{
Andrea Scotti $^{1}$ (D) $\cdot$ Andrea Munafò $^{2}$ (D) $\cdot$ Alberto Margonato $^{3} \cdot$ Cosmo Godino $^{3}$ (D)
}

Accepted: 13 July 2021 / Published online: 22 July 2021

(c) The Author(s) 2021

\begin{abstract}
A severe secondary mitral regurgitation (SMR) can be found in a significant portion of patients affected by advanced heart failure (AHF). Conventional therapies (optimal medical therapy, devices, surgery) present restricted clinical efficacy in this stage of the left ventricle disease which is burdened by high mortality and morbidity rates. Although the treatment of choice is represented by heart transplantation (HTx), there is an unmet need related to the limited supply of donor hearts (as opposed to the growing prevalence of AHF) and the low eligibility of highly symptomatic patients. In case of concomitant severe SMR, transcatheter mitral valve therapies (repair and replacement) may play a crucial role in this setting. While a direct prognostic improvement after correction of SMR has yet to be proved, AHF patients can benefit from the following: hemodynamic stabilization, symptomatic relief, normalization of pulmonary arterial pressures, and reduction in hospitalizations for acute heart failure. Obtaining these results may lead to the clinical consequences of reaching the HTx in good enough clinical status (bridge to heart transplantation), becoming eligible for the HTx (bridge to HTx candidacy), and being delisted for clinical improvement (bridge to recovery). Therefore, achieving traditional secondary endpoints in patients with AHF and SMR can translate into significant clinical implications.
\end{abstract}

Keywords Mitral regurgitation - Advanced heart failure $\cdot$ Transcatheter mitral valve repair $\cdot$ Transcatheter mitral valve replacement $\cdot$ Heart transplantation

\section{Introduction}

The burden of heart valve diseases represents a major public health problem. In the developed countries, mitral regurgitation is the most common valve disorder with a prevalence increasing with age [1]. Particularly, due to the increased number of patients with left sided heart failure, secondary mitral regurgitation (SMR), a common phenomenon in this scenario, has become the most frequent form.

The latter constitutes both a diagnostic and a therapeutic challenge. Up to $24 \%$ of patients with SMR are affected by

Andrea Scotti

a.scotti@ hotmail.com

1 Department of Cardiac Thoracic Vascular Sciences and Public Health, University of Padua Medical School, Via Giustiniani 2, 35128 Padua, Italy

2 Division of Cardiology, IRCCS Policlinico San Matteo Foundation, Pavia, Italy

3 Division of Cardiology, San Raffaele Scientific Institute, Milan, Italy heart failure due to left ventricular (LV) dysfunction leading to an underestimation of the mitral valve disease itself $[2$, 3]. Since $L V$ disease is the most important prognostic factor, guideline-directed medical therapies (GDMT) for heart failure play a major role for these patients. However, SMR is not a merely bystander but contributes to worsening survival and LV function [4-6]. This vicious cycle may lead to a stage of advanced heart failure (AHF) where patients no longer respond to conventional therapies. While the correction of SMR has yet to prove its effectiveness in terms of survival, it has been shown how it can translate into significant clinical implications for AHF patients.

\section{Advanced heart failure}

Chronic heart failure is a common condition among adults, with an increasing prevalence because of the aging of the population and improved cardiovascular treatments (especially for acute events). Although it is a heterogeneous syndrome, all etiologies share a final common pathway 
with progressive worsening of symptoms and cardiac function. The final stage when conventional therapies (GDMT, implanted devices, surgery) are no more effective is represented by the AHF, clinically diagnosed on the basis of patient's symptoms, cardiac dysfunction, functional capacity indices, and prognostic markers. The low incidence and the evolving definitions of AHF make it difficult to estimate its prevalence. Data from ADHERE (Acute Decompensated Heart Failure) national registry of $\sim 23,000$ hospitalized patients report a prevalence of AHF close to 5\% [7]. Since the AHF is burdened by high mortality and morbidity rates, early recognition of this stage of the disease is of paramount importance. Indeed, the heart failure association of the European Society of Cardiology stresses the importance of a timely referral of patients with AHF to tertiary hub centers where the broad spectrum of advanced therapies can be offered [8]. The eligibility for such specialized care can be verified with the useful mnemonic "I Need Help" consisting of inotropes, New York Heart Association (NYHA) class II/ III or elevated natriuretic peptides, renal or liver dysfunction, very low ejection fraction $(<20 \%)$, recurrent appropriate defibrillator shocks, hospitalizations for heart failure in the previous 12 months, edema or increasing diuretic doses, low blood pressure (systolic $<90$ to $100 \mathrm{mmHg}$ ), and inability to up-titrate or maintain prognostic drugs [9].

\section{Therapies for advanced heart failure}

The treatment of choice for most patients with AHF is represented by heart transplantation (HTx). To date, the complications related to infectious diseases, immunosuppression, and donor/recipient selection have been significantly reduced. As a result, survival, quality of life, and exercise tolerance increased. The main limitations of this treatment consist in the limited availability of donor hearts and the poor eligibility of patients who are often in such a compromised stage that no benefit can be expected. While the number of transplants appears to have reached a plateau, the HTx waiting lists are persistently expanding. Prolonged (over 12 months) times on waiting lists are associated with mortality rates ranging between $13 \%$ at 1 year and $19 \%$ up to 3 years [10].

Long-term mechanical circulatory support (MCS) obtained through left ventricular assist device (LVAD) implantation represents a successfully therapeutic solution for this population. The Risk Assessment and Comparative Effectiveness of Left Ventricular Assist Device and Medical Management in Ambulatory Heart Failure Patients (ROADMAP) study provided the risk-benefit ratio for a shared decision on elective LVAD therapy for AHF. Higher survival with improved functional status, improved quality of life, and reduced depression favored LVAD over optimal medical therapy; on the other hand, LVAD implantation caused more hospitalizations for heart failure and major adverse events (bleedings, arrhythmias) that reduced after the first year of follow-up [11].

LVAD placement is now indicated for AHF patients (1) not eligible for HTx (LVAD as destination therapy, actually the main application worldwide), (2) with reversible contraindications to HTx (e.g., obesity, pulmonary arterial hypertension, or renal failure) whose resolution could establish the feasibility of HTx (bridge to candidacy, Class IIb, Level of Evidence: C) [12], or (3) with the possibility of cardiac function recovery (bridge to recovery). Moreover, (4) AHF patients who are still on the HTx waiting list, could undergo LVAD implantation in order to keep them alive without worsening hemodynamics until a donor organ becomes available (bridge to HTx) [13, 14].

In the wide spectrum of AHF patients, LVAD is still reserved for those with an extremely advanced clinical condition (identified as patients with an INTERMACS profile of 1 to 3) [14]. Besides, LVAD implantation has an inherent risk of serious adverse events, whose perceived impact has even more value in patients with prohibitive operative risk, limited life expectancy, and severe psychosocial limitations. As a result, a significant portion of them is not eligible for MCS or refuses this therapy for several personal reasons.

Finally, according to the fluctuating and progressive history of the disease, AHF patients might require short term MCS due to sudden worsening of their clinical condition. Among the percutaneous options, there are four established devices including Impella (Abiomed, Danvers, MA), TandemHeart (CardiacAssist, Pittsburgh, PA), extracorporeal membrane oxygenation, and intra-aortic balloon pump. All of them might guarantee hemodynamics and end-organ perfusion stabilization, allowing patients to be eligible for HTx or long-term MCS.

\section{Secondary mitral regurgitation}

Hemodynamically significant SMR is a common finding in patients affected by AHF: severe or moderate-severe SMR in about $15 \%$ and moderate or worse SMR in about $40 \%$ [15]. The severity of SMR is associated with the degree of LV dilatation, systolic/diastolic LV dysfunction, and pulmonary hypertension, resulting in an exacerbation of heart failure symptoms.

In heart failure with reduced ejection fraction, SMR usually develops as a "ventricular-secondary" mechanism: a competent coaptation of mitral valve leaflets is impaired by mitral annulus dilation and papillary muscles displacement due to LV dilatation. The common coexistence of left atrial dilatation and/or atrial fibrillation results in the elevation of atrial pressures and dilatation of mitral annulus that worsen the effects and prognosis of SMR with an "atrial-secondary" mechanism $[16,17]$. This is the rationale 
for the improvement of SMR after GDMT for heart failure (ventricular-secondary) and rhythm/rate control for atrial fibrillation (atrial-secondary).

When AHF symptoms and SMR severity no longer respond to conventional heart failure therapies, it is likely that AHF has evolved to valvular-AHF. In this context, mitral valve intervention may play a key role in patients' treatment by ending this vicious cycle. Obtaining a hemodynamic stabilization could enable the up-titration of GDMT and provide more time to reach advanced therapies. Considering the association of AHF and high/prohibitive surgical risk, the reduction of SMR could be addressed in most AHF patients with a transcatheter solution $[18,19]$.

\section{Transcatheter mitral valve repair}

The available transcatheter mitral valve repair (TMVR) techniques for SMR (Fig. 1) [20] can be classified as follows:

- Edge-to-edge repair = MitraClip, PASCAL
- Percutaneous annuloplasty $=$ AccuCinch, ARTO, Carillon, Cardioband, Millipede, Mitral Loop Cerclage, Mitralign

The MitraClip (Abbott Laboratories, Menlo Park, CA, USA) device is a clip that reproduces the surgical Alfieristitch by grasping the mitral valve leaflets through its arms and providing a more competent coaptation. The PASCAL (Edwards Lifesciences, Irvine, CA) system adopts the same rationale of the MitraClip with the addition of a $10-\mathrm{mm}$ central spacer within the regurgitant office to reduce the tension on the valve leaflets caused by the traction paddles. Favorable features are represented by a grasping length $>10 \mathrm{~mm}$, a coaptation length $\geq 2 \mathrm{~mm}$, and a coaptation depth $\leq 11 \mathrm{~mm}$ [21]. Suboptimal results may be expected in the presence of leaflets' perforations, calcifications on the grasping zone, or deep cleft-like indentations. Pre-procedural mitral valve area $\leq 3 \mathrm{~cm}^{2}$ and mean mitral valve gradient $>4 \mathrm{mmHg}$ need to be excluded to prevent a procedural failure related to intractably high transmitral gradients [22].
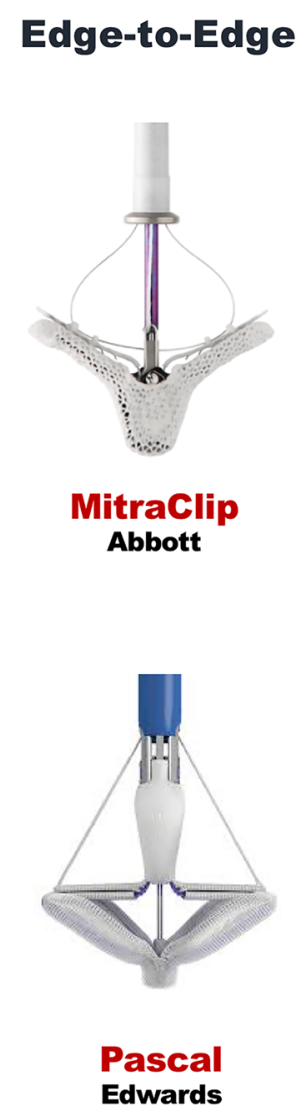

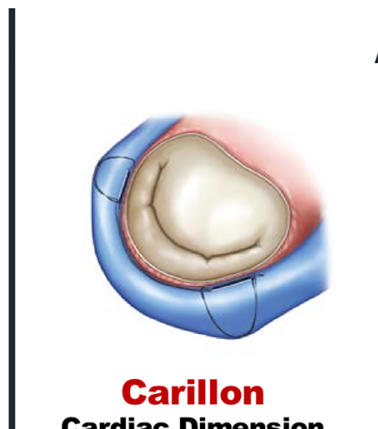

Cardiac Dimension
Indirect Annuloplasty
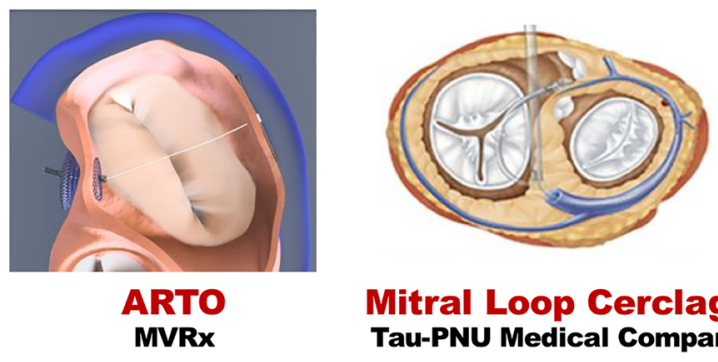

Mitral Loop Cerclage Tau-PNU Medical Company

\section{Direct Annuloplasty}

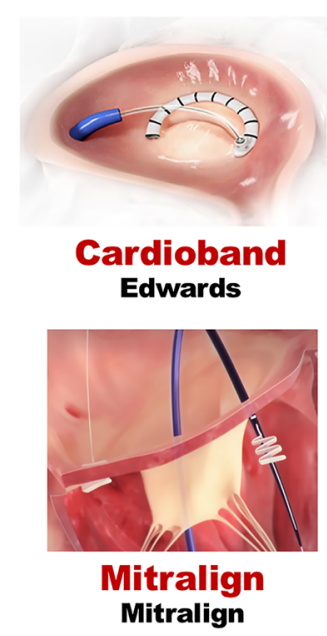

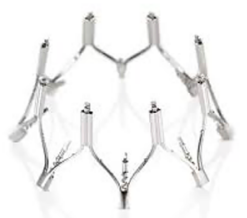

Millipede Boston

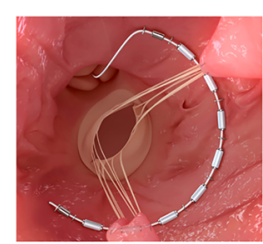

AccuCinch ANCORA Heart

Fig. 1 Transcatheter mitral valve repair devices. Reproduced from Vessel Plus 2021;5:6 [20] 
Percutaneous annuloplasty is the solution to mitral annulus dilatation, whose narrowing allows to approximate the valve leaflets and reduce the orifice area. The direct approach (Cardioband, Millipede, Mitralign, AccuCinch) can be performed by a transseptal or retrograde LV access and consists of implanting a variable number of anchors around the mitral annulus and tether them with the delivery system. Indirect annuloplasty devices (Carillon, ARTO, Mitral Loop Cerclage Catheter System) promote a positive remodeling by applying a traction force on the coronary sinus. The main limitations of these procedures rely on their complexity and long duration, unfavorable anatomy of the coronary sinus and its branch veins, and the risk of coronary compression. An angio-CT is mandatory in the screening phase to exclude the presence of calcifications, the risky proximity of the left circumflex artery, and tricky anatomies. When suboptimal results are expected, percutaneous annuloplasty can be performed as a complimentary technique to edge-to-edge repair [23].

\section{Clinical evidence}

The reduction of SMR in AHF patients provides clinical and hemodynamic benefits that result in less severe symptoms, fewer heart failure hospitalizations, and, in some cases, LV remodeling (Fig. 2). The clinical implications of these outcomes rely on the presence of irreversible contraindications to HTx. If this condition occurs, the purpose of SMR reduction consists in providing an improved quality of life and enabling the up-titration of GDMT. In AHF patients who are potentially eligible for HTx (absent or reversible contraindications), the rationale for treating SMR can be classified into the following strategies:

- Bridge to heart transplantation (BTT)

- Bridge to HTx candidacy (BTC)

- Bridge to recovery (BTR)
Of all the TMVR systems, the MitraClip is the only one that has strong evidence for clinical efficacy, with the other devices having limited system numbers or being no longer available. In 2011, Franzen and colleagues were the first to report the feasibility and effectiveness of MitraClip implantation in their cohort of 50 AHF patients; despite an expected significant mortality rate (6\% at 30 days and $19 \%$ at 6 months), the reduction of SMR severity was accompanied by a clinical improvement expressed as NYHA functional class, 6-min walk distance, LV volumes, and NT-proBNP plasma levels [24]. Further analysis found a positive impact of TMVr in terms of lower pulmonary artery pressures and resistances, with the consequence of including patients previously ineligible for this therapy on the HTx list (BTC) [25, 26]. The first results of the feasibility of a BTT strategy were described after the implantation of MitraClip alone or in combination with the Carillon device (Cardiac Dimensions, Kirkland, WA, USA) [27, 28]. The achievement of great clinical and hemodynamic benefits can even lead to recovery such as removing the patient from the HTx list (BTR) [29].

The "MitraBridge" study is an international, multicenter registry that collected data on MitraClip implantation in 119 AHF patients with severe or moderate-to-severe SMR [30]. The aim of the investigators was to analyze the outcomes of TMVr as a bridge strategy in this extreme setting of AHF patients. Baseline characteristics depict a clearly different population from those of published trials ( $80 \%$ of patients were formally ineligible according to the Clinical Outcomes Assessment of the MitraClip Percutaneous Therapy [COAPT] trial criteria) [31]: median age was 58 years, almost all of them (95\%) were in NYHA class III - IV, severely depressed LV function (ejection fraction with a median of $26 \%$ and below $30 \%$ in $71.5 \%$ of patients), an abnormal cardiac index (median of $1.9 \mathrm{~L} / \mathrm{min} / \mathrm{m}^{2}$ and below $2 \mathrm{~L} / \mathrm{min} / \mathrm{m}^{2}$ in $60 \%$ of patients), and $40.5 \%$ of them had an INTERMACS profile of 1 to 4 . Reported results
Fig. 2 Expected outcomes after transcatheter mitral valve repair in patients with advanced heart failure and secondary mitral regurgitation. *Published evidences on MitraClip implantation. GDMT, guideline directed medical therapy; HF, heart failure; HTx heart transplantation; LVEDV, left ventricular end diastolic volume; NT-proBNP, $\mathrm{N}$-terminal pro-brain natriuretic peptide; NYHA, New York Heart Association; 6MWT, 6-min walk distance

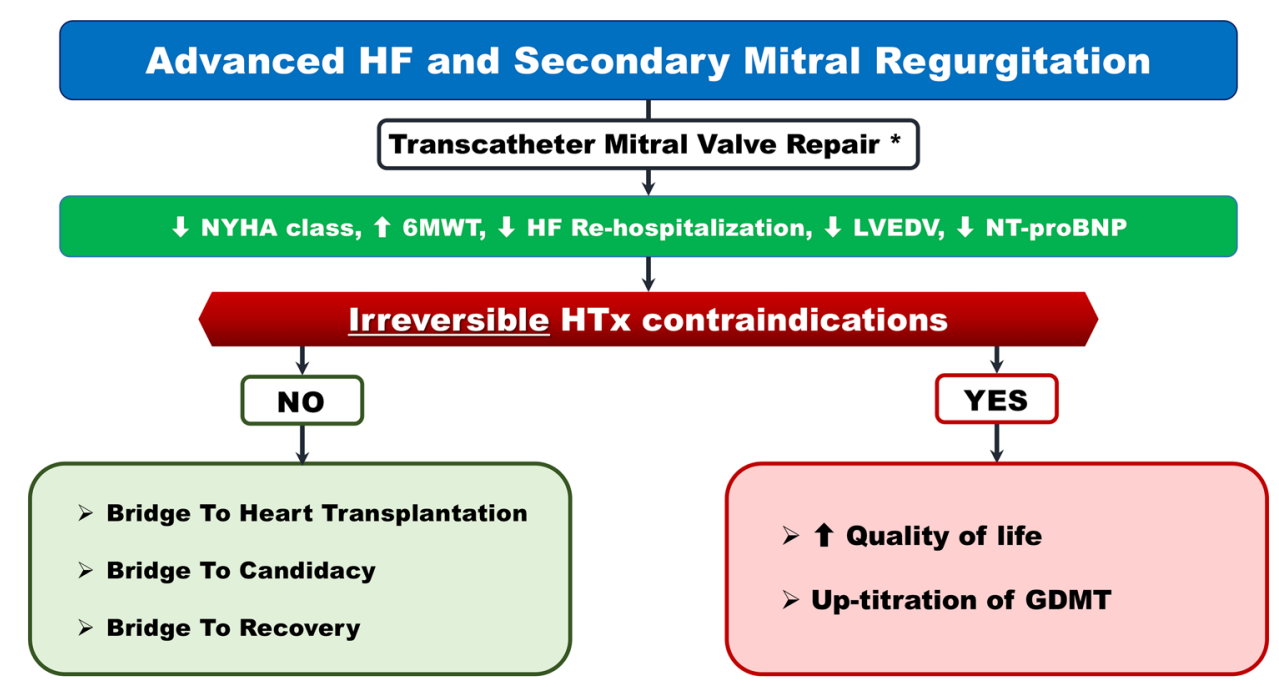


showed that MitraClip implantation as a bridge strategy was safe with a procedural success of $87.5 \%$ and no deaths at 30 days. One-year outcomes found two-thirds of patients free from the composite endpoint (all-cause death, urgent HTx or LVAD implantation, first rehospitalization for heart failure), $15.5 \%$ became eligible for HTx (BTC), and nearly a quarter of them (27 patients) were removed from consideration for HTx because of clinical improvement (BTR). These exploratory results are promising and provide a proof of concept of MitraClip implantation in patients with AHF who are on the waiting list or at that moment not eligible or at high risk for HTx.

\section{Transcatheter mitral valve replacement}

Transcatheter mitral valve replacement (TMVRpl) is a promising technology that can provide predictable and reproducible results. Currently, only limited specialized centers can perform this technique, whose evidence is still too scarce to be as widespread as TMVr.

The available TMVRpl devices are trileaflet biological valves built with xenogenic leaflets that are mounted on self-expanding frames (Fig. 3) [20]. Transcatheter valve implantation on the mitral valve is not straightforward as its counterpart on the aortic valve due to complex mitral anatomy characterized by a peculiar D-shape and a wellrepresented sub-valvular apparatus. The conformation of the native valve hinders the generation of a uniform radial force with the risk of paravalvular leakage and prosthesis migration. The approach of some companies to hold the valve in place is the application of anchors to the annulus, the subvalvular apparatus, or the left ventricular apex. The risk of paravalvular leakage may only be partially decreased with the over-expansion and circularization of the valve, because of the potential obstruction of the left ventricular outflow tract. This dreaded complication also occurs in the case of mitral aortic angle $<120^{\circ}$, severe LV septal hypertrophy, extremely wide annulus, and too ventricular implant sites. Most of the systems under investigation require transapical access that enables the delivery of large devices and allows for better coaxiality with the native valve.

\section{Clinical evidence}

TMVRpl experience in native mitral valves is still limited to trials with slightly more than 500 procedures. The Tendyne (Abbott Vascular; Santa Clara, CA) has been the first transcatheter mitral valve implantation system to be approved in Europe in 2020 and, to date, no device has gained the Food and Drug Administration approval for the USA. The analysis of the first 308 TMVRpls shows that the procedure is feasible and, compared to the COAPT trial, provides better results in terms of post-procedural mitral regurgitation reduction (moderate or severe in $1.5 \%$ vs. $7.3 \%$, respectively), symptomatic improvement (NYHA class III or IV $14.4 \%$ vs. $20.3 \%$, respectively), and hospitalizations for heart failure ( $26.4 \%$ vs. $35.7 \%$, respectively) [31, 32]. However, the rates of complications (stroke, endocarditis, and unplanned mitral valve surgery) and mortality (13.6\% at 30 days, $27.6 \%$ at a mean follow-up of 10 months) are still unacceptable. It is necessary to specify that these data come from studies that investigated different TMVRpl systems and included very heterogeneous patients: from the elderly with a relevant comorbidity burden and very high operative risk (compassionate use) to those who were fitter and passed a selective enrollment screening. However, the improvements that have been achieved over time are promising. A real-world series of 11 patients treated with Tiara and Tendyne systems reported a $100 \%$ technical success with no procedural or in-hospital mortality [33]. Recent data on
Fig. 3 Transcatheter mitral valve replacement devices. Reproduced from Vessel Plus 2021;5:6 [20]

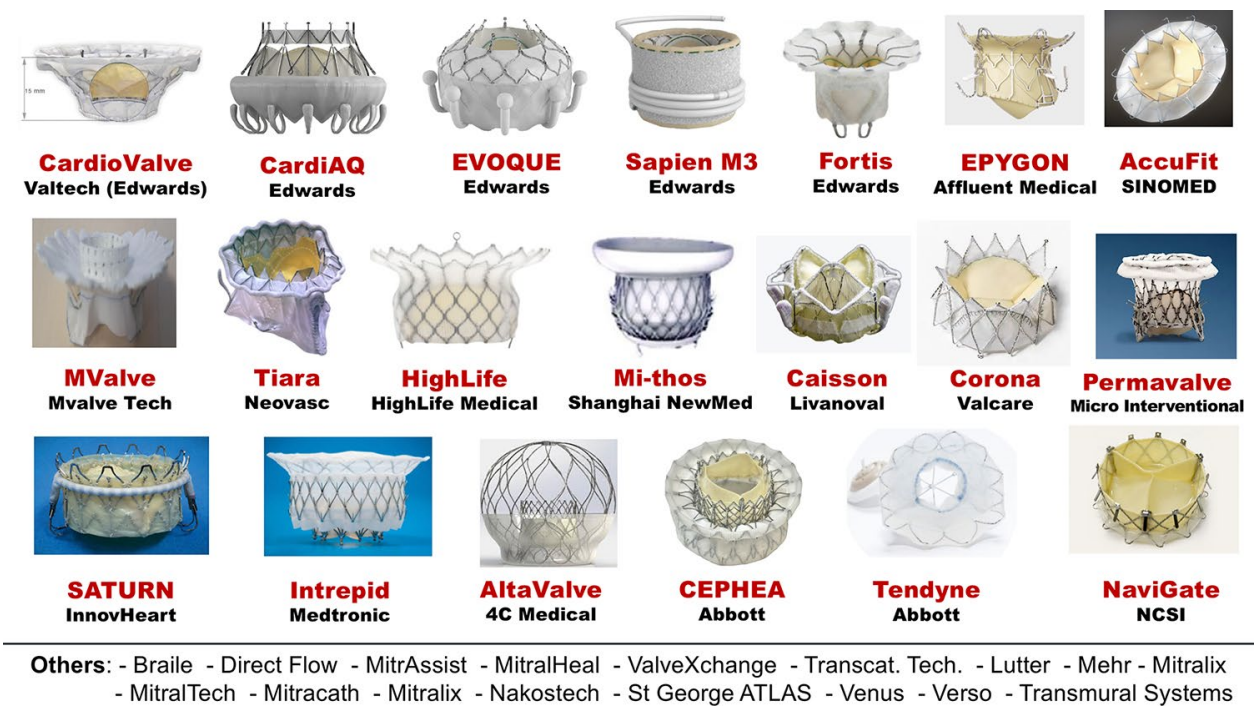


TMVRpl with new-generation devices who are currently under clinical evaluation (2 AltaValve, 5 CardioValve, 4 Cephea, 14 Evoque, 15 Highlife, 50 Interpid, 45 M3, 109 Tendyne, 79 Tiara) show a weighted mean technical success of $94 \%$, postprocedural mitral valve mean gradient of 3.7 (3.5-3.8), at least moderate residual MR in $3.1 \%$ of cases, and procedural and 30-day mortality rates of $1.4 \%$ and $9.5 \%$, respectively [34].

TMVRpl for severe SMR in patients at high surgical risk and previous mitral surgery (bioprosthesis or annuloplasty) or severe mitral annular calcification (valve-in-valve [ViV], valve-in-ring [ViR], and valve-in-MAC, respectively) can be performed with the transcatheter aortic SAPIEN XT and S3 (Edwards Lifesciences, Irvine, CA), the MyVal (Meril, Vapi, India), or the transcatheter pulmonary Melody (Medtronic, Minneapolis, MN) valve [35-37]. Data from the VIVID (Valve-in-Valve International Data) registry on mitral ViV and ViR procedures identify the SAPIEN 3 as the most frequently used device $(\sim 42 \%)$ and the transapical as an access route more commonly used in ViV than in ViR (64\% vs. $51 \%$ ). In terms of procedural results, MVARC-defined technical success ranges from $82(\mathrm{ViR})$ to $93.5 \%(\mathrm{ViV})$ and MVARC-defined device success from $32 \%$ (ViR) to $39.4 \%$ (ViV). The most common cause of device failure can be identified in postprocedural mean gradient $\geq 5 \mathrm{mmHg}(96 \%$ $\mathrm{ViV}, 88 \% \mathrm{ViR})$. In addition to lower success rates, $\mathrm{ViR}$ was burdened by higher rates of malposition, increased need for second transcatheter valve implantation, and higher rates of LVOT obstruction. These procedural outcomes are accompanied by a higher 1-year mortality for ViR (23.2\% vs. $13.8 \%$, ViR vs. ViV, respectively) that persists at 4 years [38]. The particularly rigid and non-circular nature of surgical rings may be the cause of worse outcomes due to more residual MR and device underexpansion.

A limitation to TMVRpl is represented by the transapical access: patients with a remodeled LV or with ischemic scars may not tolerate well this approach. As they are developed, systems that deliver the valve with a transseptal route through transfemoral introducers are expected to result in fewer complications and better outcomes.

Valve durability after TMVRpl is another great concern that needs to be better elucidated. The age of patients undergoing TMVRpl (about 10 years younger than recipients of aortic valves) plays an important role in the rate of structural valve degeneration [39]. Enhanced hemodynamic shear stress, the difference in calcium metabolism, patient prosthesis mismatch, and residual leaflet antigenicity expose young patients to an accelerated valve deterioration [40]. Another key factor of this process is represented by the systolic pressure gradient that produces greater mechanical stress on mitral bioprosthesis if compared to the aortic ones.

A relevant risk of valve thrombosis undermines TMVRpl: 6\% to 8\% after the implantation of Tendyne (Abbott Vascular, Abbott Park, IL), Fortis (Edwards Lifesciences, Irvine,
CA), and HighLife (HighLife Medical, Irvine, CA) device [41-43]. The adoption of oral anticoagulation in addition to antiplatelet therapy resulted in no thrombotic complications at 1 year, at the expense of a significant rate (18\%) of 30-day major bleedings after intrepid valve (Medtronic Inc., Redwood City, CA) implantation [44]. Taking the cue from the recommendations after surgical mitral valve replacement, an anticoagulation strategy for the first 3 months seems reasonable to protect against early valve thrombosis [19]. After this period, when endothelization is complete, a serial clinical and imaging follow-up should guide toward a prompt diagnosis and treatment of any late thrombotic complication.

\section{Future perspectives}

At present, there are limited exploratory studies on the transcatheter treatment of SMR in patients with AHF. While a direct prognostic improvement after correction of SMR has yet to be proved, AHF patients can greatly benefit from the following: hemodynamic stabilization, symptomatic relief, normalization of pulmonary arterial pressures, and reduction in hospitalizations for acute heart failure. Obtaining these results may lead to the clinical consequences of reaching the HTx in good enough clinical status (BTT), becoming eligible for the HTx (BTC), and being delisted for clinical improvement (BTR). Therefore, achieving "not-so-hard endpoints" in patients with AHF and SMR can translate into great clinical implications.

More studies are needed to investigate who more likely will benefit the most from SMR treatment at this advanced stage of LV disease. Since left and right ventricular reverse remodeling have been found as turning points of favorable outcomes, it should be assessed whether this phenomenon can also occur in this population $[45,46]$.

The lack of sufficient evidence and the lesson learned from the surgical experience make TMVr preferable over TMVRpl [21]. However, as more studies on TMVRpl are completed, this technology could gain much appeal and be considered a viable alternative to TMVr instead of being regarded as a second choice. TMVRpl devices can provide more predictable results in terms of MR reduction, require a less-technically demanding implantation, and have the potential to be able to adapt to such different anatomies, including those that represent exclusion criteria for TMVr (multisegment or commissural disease, perforations, and clefts).

\section{Conclusions}

Transcatheter therapies for SMR may play an important role in AHF patients. Exploratory studies proved the procedural safety and clinical efficacy (BTC, BTT, and BTR) 
of this treatment. Further investigations are needed to better characterize AHF patients with concomitant SMR, identify optimal candidates for transcatheter therapies, and understand the consequences of the different bridge strategies. While TMVr has more evidence in this setting, TMVRpl is expected to become a valid alternative in the near future. Having a complete mitral toolbox will be the key to handle all the anatomies and provide a patienttailored approach.

Funding Open access funding provided by Università degli Studi di Padova within the CRUI-CARE Agreement.

\section{Declarations}

Conflict of interest The authors declare no competing interests.

Open Access This article is licensed under a Creative Commons Attribution 4.0 International License, which permits use, sharing, adaptation, distribution and reproduction in any medium or format, as long as you give appropriate credit to the original author(s) and the source, provide a link to the Creative Commons licence, and indicate if changes were made. The images or other third party material in this article are included in the article's Creative Commons licence, unless indicated otherwise in a credit line to the material. If material is not included in the article's Creative Commons licence and your intended use is not permitted by statutory regulation or exceeds the permitted use, you will need to obtain permission directly from the copyright holder. To view a copy of this licence, visit http://creativecommons.org/licenses/by/4.0/.

\section{References}

1. Nkomo VT, Gardin JM, Skelton TN et al (2006) Burden of valvular heart diseases: a population-based study. Lancet 368:10051011. https://doi.org/10.1016/S0140-6736(06)69208-8

2. Iung B, Vahanian A (2011) Epidemiology of valvular heart disease in the adult. Nat Rev Cardiol 8:162-172. https://doi.org/10. 1038/nrcardio.2010.202

3. Rossi A, Dini FL, Faggiano P et al (2011) Independent prognostic value of functional mitral regurgitation in patients with heart failure. A quantitative analysis of 1256 patients with ischaemic and non-ischaemic dilated cardiomyopathy. Heart 97:1675-1680. https://doi.org/10.1136/hrt.2011.225789

4. Sannino A, Smith RL, Schiattarella GG et al (2017) Survival and cardiovascular outcomes of patients with secondary mitral regurgitation: a systematic review and meta-analysis. JAMA Cardiol 2:1130-1139. https://doi.org/10.1001/jamacardio.2017.2976

5. Goliasch G, Bartko PE, Pavo N et al (2018) Refining the prognostic impact of functional mitral regurgitation in chronic heart failure. Eur Heart J 39:39-46. https://doi.org/10.1093/eurheartj/ ehx402

6. Godino C, Scotti A, Munafò A et al (2018) Observed versus predicted mortality after MitraClip treatment in patients with symptomatic heart failure and significant functional mitral regurgitation. Eur J Heart Fail 20:1495-1496

7. Costanzo MR, Mills RM, Wynne J (2008) Characteristics of "Stage D" heart failure: insights from the Acute Decompensated Heart Failure National Registry Longitudinal Module (ADHERE
LM). Am Heart J 155:339-347. https://doi.org/10.1016/j.ahj. 2007.10.020

8. Crespo-Leiro MG, Metra M, Lund LH et al (2018) Advanced heart failure: a position statement of the Heart Failure Association of the European Society of Cardiology. Eur J Heart Fail 20:15051535. https://doi.org/10.1002/ejhf.1236

9. Baumwol J (2017) "I Need Help"-a mnemonic to aid timely referral in advanced heart failure. J Hear Lung Transplant 36:593594. https://doi.org/10.1016/j.healun.2017.02.010

10. Eurotransplant International Foundation (2019) annual report. Available at the following website: https://www.eurotransplant. org/wp-content/uploads/2020/06/Annual-Report-2019.pdf

11. Starling RC, Estep JD, Horstmanshof DA et al (2017) Risk assessment and comparative effectiveness of left ventricular assist device and medical management in ambulatory heart failure patients: the ROADMAP study 2-year results. JACC Hear Fail 5:518-527. https://doi.org/10.1016/j.jchf.2017.02.016

12. Mehra MR, Canter CE, Hannan MM et al (2016) The 2016 International Society for Heart Lung Transplantation listing criteria for heart transplantation: a 10-year update. J Hear Lung Transplant 35:1-23. https://doi.org/10.1016/j.healun.2015.10.023

13. Ponikowski P, Voors AA, Anker SD et al (2016) 2016 ESC guidelines for the diagnosis and treatment of acute and chronic heart failure. Eur Heart J 37:2129-2200m. https://doi.org/10.1093/ eurheartj/ehw128

14. Goldstein DJ, Meyns B, Xie R et al (2019) Third annual report from the ISHLT mechanically assisted circulatory support registry: a comparison of centrifugal and axial continuous-flow left ventricular assist devices. J Hear Lung Transplant 38:352-363. https://doi.org/10.1016/j.healun.2019.02.004

15. Patel JB, Borgeson DD, Barnes ME et al (2004) Mitral regurgitation in patients with advanced systolic heart failure. J Card Fail 10:285-291. https://doi.org/10.1016/j.cardfail.2003.12.006

16. Lancellotti P, Garbi M (2018) Progression of secondary mitral regurgitation: from heart failure to valvular heart failure. Eur Heart J Cardiovasc Imaging 19:613-614. https://doi.org/10.1093/ ehjci/jey040

17. Godino C, Sisinni A, Pivato CA et al (2020) Prognostic value of pre-operative atrial fibrillation in patients with secondary mitral regurgitation undergoing MitraClip implantation. Am J Cardiol. https://doi.org/10.1016/j.amjcard.2020.12.043

18. Otto CM, Nishimura RA, Bonow RO et al (2021) 2020 ACC/AHA guideline for the management of patients with valvular heart disease. J Am Coll Cardiol. https://doi.org/10.1016/j.jacc.2020.11.018

19. Baumgartner H, Falk V, Bax JJ et al (2017) 2017 ESC/EACTS guidelines for the management of valvular heart disease. Eur Heart J 38:2739-2786. https://doi.org/10.1093/eurheartj/ehx391

20. Scotti A, Galasso M, Margonato A, Godino C (2021) Patient selection for trans-catheter mitral valve repair versus replacement: ongoing indications and glimpse to the future. Vessel Plus. https:// doi.org/10.20517/2574-1209.2020.68

21. Taramasso M, Gavazzoni M, Nickenig G, Maisano F (2019) Transcatheter mitral repair and replacement: which procedure for which patient? EuroIntervention 15:867-874. https://doi.org/10. 4244/eij-d-19-00743

22. Lubos E, Schlüter M, Vettorazzi E et al (2014) MitraClip therapy in surgical high-risk patients: identification of echocardiographic variables affecting acute procedural outcome. JACC Cardiovasc Interv 7:394-402. https://doi.org/10.1016/j.jcin.2013.12.198

23. Braun D, Näbauer M, Massberg S, Hausleiter J (2019) One-stop shop: simultaneous direct mitral annuloplasty and percutaneous mitral edge-to-edge repair in a patient with severe mitral regurgitation. Catheter Cardiovasc Interv 93:E318-E319. https://doi.org/ $10.1002 / \mathrm{ccd} .27848$ 
24. Franzen O, van der Heyden J, Baldus S et al (2011) MitraClip therapy in patients with end-stage systolic heart failure. 569-576. https://doi.org/10.1093/eurjhf/hfr029

25. Geis NA, Pleger ST, Bekeredjian R et al (2018) Haemodynamic effects of percutaneous mitral valve edge-to-edge repair in patients with end-stage heart failure awaiting heart transplantation. ESC Hear Fail 5:892-901. https://doi.org/10.1002/ehf2.12313

26. Crimi G, Gritti V, Ghio S et al (2018) MitraClip procedure as 'bridge to list', the ultimate therapeutic option for end-stage heart failure patients not eligible for heart transplantation due to severe pulmonary hypertension. Pulm Circ 8. https://doi.org/10.1177/ 2045894018791871

27. Garatti A, Castelvecchio S, Bandera F et al (2015) Mitraclip procedure as a bridge therapy in a patient with heart failure listed for heart transplantation. Ann Thorac Surg 99:1796-1799. https://doi. org/10.1016/j.athoracsur.2014.07.079

28. Sankar NM, Ramani SS, Anantharaman R, Cherian KM (2018) MitraClip and mitral annuloplasty device as a bridge to transplantation. Asian Cardiovasc Thorac Ann 26:57-59. https://doi.org/ $10.1177 / 0218492317738385$

29. Godino C, Scotti A, Agricola E et al (2017) Young patient with advanced heart failure no longer a candidate for heart transplantation after MitraClip ${ }^{\circledR}$ procedure. J Heart Valve Dis 26:234-236

30. Godino C, Munafò A, Scotti A et al (2020) MitraClip in secondary mitral regurgitation as a bridge to heart transplantation: 1-year outcomes from the International MitraBridge Registry. J Hear Lung Transplant 39:1353-1362. https://doi.org/10.1016/j. healun.2020.09.005

31. Stone GW, Lindenfeld JA, Abraham WT et al (2018) Transcatheter mitral-valve repair in patients with heart failure. N Engl J Med 379:2307-2318. https://doi.org/10.1056/NEJMoa1806640

32. Del Val D, Ferreira-Neto AN, Wintzer-Wehekind J et al (2019) Early experience with transcatheter mitral valve replacement: a systematic review. J Am Heart Assoc 8:e013332. https://doi.org/ 10.1161/JAHA.119.013332

33. Ludwig S, Kalbacher D, Schofer N et al (2021) Early results of a real-world series with two transapical transcatheter mitral valve replacement devices. Clin Res Cardiol 110:411-420. https://doi. org/10.1007/s00392-020-01757-z

34. Alperi A, Granada JF, Bernier M et al (2021) Current status and future prospects of transcatheter mitral valve replacement. J Am Coll Cardiol 77:3058-3078. https://doi.org/10.1016/j.jacc.2021. 04.051

35. De Weger A, Ewe SH, Delgado V, Bax JJ (2011) First-in-man implantation of a trans-catheter aortic valve in a mitral annuloplasty ring: novel treatment modality for failed mitral valve repair. Eur J Cardio-thoracic Surg 39:1054-1056. https://doi.org/10. 1016/j.ejcts.2010.09.021
36. Schaefer A, Conradi L, Seiffert M et al (2016) Valve-in-valve procedures in failing biological xenografts using a novel balloonexpandable device: experience in aortic, mitral, and tricuspid positions. Thorac Cardiovasc Surg 64:366-373. https://doi.org/ $10.1055 / \mathrm{s}-0035-1558647$

37. Ielasi A, Buono A, Medda M et al (2020) Trans-catheter valvein-valve implantation with a novel balloon expandable device in patients with bioprosthetic heart valve failure: a case series. Cardiovasc Revascularization Med. https://doi.org/10.1016/j.carrev. 2020.11 .018

38. Simonato M, Whisenant B, Ribeiro HB et al (2021) Transcatheter mitral valve replacement after surgical repair or replacement comprehensive midterm evaluation of valve-in-valve and valve-in-ring implantation from the VIVID registry. Circulation 143:104-116. https://doi.org/10.1161/CIRCULATIONAHA.120.049088

39. Brown JM, O'Brien SM, Wu C et al (2009) Isolated aortic valve replacement in North America comprising 108,687 patients in 10 years: changes in risks, valve types, and outcomes in the Society of Thoracic Surgeons National Database. J Thorac Cardiovasc Surg 137:82-90. https://doi.org/10.1016/j.jtcvs.2008.08.015

40. Mylotte D, Andalib A, Thériault-Lauzier P et al (2015) Transcatheter heart valve failure: a systematic review. Eur Heart J 36:1306-1327. https://doi.org/10.1093/eurheartj/ehu388

41. Sorajja P, Moat N, Badhwar V et al (2019) Initial feasibility study of a new transcatheter mitral prosthesis: the first 100 patients. J Am Coll Cardiol 73:1250-1260. https://doi.org/10.1016/j.jacc. 2018.12.066

42. Piazza N (2018) Transcatheter mitral valve replacement: HighLife. In: EuroPCR. Paris, France

43. Regueiro A, Ye J, Fam N et al (2017) 2-year outcomes after transcatheter mitral valve replacement. JACC Cardiovasc Interv 10:1671-1678. https://doi.org/10.1016/j.jcin.2017.05.032

44. Bapat V, Rajagopal V, Meduri C et al (2018) Early experience with new transcatheter mitral valve replacement. J Am Coll Cardiol 71:12-21. https://doi.org/10.1016/j.jacc.2017.10.061

45. Adamo M, Godino C, Giannini C et al (2018) Left ventricular reverse remodelling predicts long-term outcomes in patients with functional mitral regurgitation undergoing MitraClip therapy: results from a multicentre registry. Eur J Heart Fail 21:ejhf.1343. https://doi.org/10.1002/ejhf.1343

46. Godino C, Salerno A, Cera M et al (2016) Impact and evolution of right ventricular dysfunction after successful MitraClip implantation in patients with functional mitral regurgitation. IJC Hear Vasc 11:90-98. https://doi.org/10.1016/j.ijcha.2016.05.017

Publisher's Note Springer Nature remains neutral with regard to jurisdictional claims in published maps and institutional affiliations. 